\title{
Effect of an Excess of Loss Reinsurance on Upper Bounds of Ruin Probabilities
}

\author{
Nguyen Quang Chung1,2 \\ ${ }^{1}$ Department of Basic Sciences, Hungyen University of Technology and Education, Hung Yen, Vietnam \\ ${ }^{2}$ Applied Mathematics and Informatics School, Hanoi University of Science and Technology, Hanoi, Vietnam \\ Email: chungkhcb@utehy.edu.vn,chungkhcb@gmail.com
}

How to cite this paper: Chung, N.Q. (2017) Effect of an Excess of Loss Reinsurance on Upper Bounds of Ruin Probabilities. Journal of Mathematical Finance, 7, 958-974.

https://doi.org/10.4236/jmf.2017.74053

Received: August 29, 2017

Accepted: November 26, 2017

Published: November 29, 2017

Copyright $\odot 2017$ by author and Scientific Research Publishing Inc. This work is licensed under the Creative Commons Attribution International License (CC BY 4.0).

http://creativecommons.org/licenses/by/4.0/

\begin{abstract}
In this paper, discrete time risk models under an excess of loss reinsurance are studied. Adjustment coefficients of the cedent and the reinsurer are established as functions of quota share level and retention level. By the martingale method, ruin probabilities of the cedent and the reinsurer still have exponential form. Finally, numerical examples are provided to illustrate the results obtained in this paper.
\end{abstract}

\section{Keywords}

Excess of Loss Reinsurance, Ruin Probability, Quota Share Level, Retention Level, Interest Rate, Markov Chain, Martingale Process

\section{Introduction}

We consider the insurer's surplus in period $n, n=1,2, \cdots$ denoted as $U_{n}$ is defined by:

$$
U_{n}=u+\sum_{i=1}^{n} Y_{i}-\sum_{i=1}^{n} X_{i}, n=1,2, \cdots
$$

where:

- $u$ is the insurer's initial surplus;

- $Y_{n}$ denotes the premium income in period $n$ (i.e., from time $n-1$ to time $n), \quad Y=\left\{Y_{n}\right\}_{n>0}$ is a sequence of independent and identically distributed (i.i.d.) non-negative random variables;

- $X_{n}$ denotes the claim amount in period $n, X=\left\{X_{n}\right\}_{n>0}$ is a sequence of i.i.d. non-negative random variables and is independent of $Y$.

The process $\left\{U_{n}\right\}_{n>0}$ defined by (1.1) is called a surplus process (see [1]). Yang [1] gave the upper bounds of ruin probabilities of the insurer by using the 
martingale method. Cai and Dickson [2] extended the surplus process in (1.1) by including the interest rate. Then, the surplus process $\left\{U_{n}\right\}_{n \geq 1}$ can be written as following

$$
U_{n}=U_{n-1}\left(1+I_{n}\right)+Y_{n}-X_{n}, \quad n=1,2, \cdots
$$

where $U_{0}=u$ and $I_{n}$ denotes the interest rate of the insurer in period $n$. The sequence $I=\left\{I_{n}\right\}_{n \geq 0}$ is assumed to be a Markov chain and independent of $X$ and $Y$. With surplus process (1.2), the upper bound of the insurer's ruin probability was established by the martingale and inductive methods in [2].

In the classical risk model, claims are assumed to be paid by one insurer. However, insurers can transfer risks from one primary insurer (the ceding company or cedent) to another one (the reinsurance company) through reinsurance contracts. For that reason, some authors extended the classical surplus process in a consideration of an excess of loss reinsurance. For example: the various articles [3] [4] [5] investigated the effect of the reinsurance contract on the upper bound of the cedent's ruin probability. The upper bounds of ruin probabilities of the cedent and the reinsurer were estimated in [6] [7] where Dam and Chung considered the risk model under quota share reinsurance. The explicit expression was given for finite-time joint survival probability of the cedent and the reinsurer in [8]. An optimal reinsurance retention was studied under ruin-related optimization criteria in [9].

This paper investigates the effect of an excess of loss reinsurance on the ultimate ruin probabilities of the cedent and the reinsurer in the discrete-time model. The risk models are investigated in two cases without interest rate and with homogeneous Markov chain interest rate. The premium income is assumed as a sequence of independent and identically distributed random variables. In particular, the author shows that for given value $\epsilon$ then there exists a quota share level and a retention level so that both the ruin probabilities of the cedent and the reinsurer are less than value $\epsilon$.

The content of this paper is organized as follows: A brief description of the models and some notions are presented in Section 2. Section 3 is devoted to the construction of the ruin-related problems in the risk model without an interest rate. The upper bounds of ruin probabilities in the risk model with interest rate are given in Section 4. Finally, numerical illustrations are given.

\section{The Risk Models}

In this paper, we investigate the effect of an excess of loss reinsurance on the surplus processes (1.1) and (1.2). First, the cedent and the reinsurer arrange an excess of loss reinsurance that we denote $\alpha(\alpha \in[0,1])$ as the quota share level and $M(M \geq 0)$ is retention level. The premiums are calculated according to the expected value principle. i.e. for each insurance company, the premium income expectation is greater than the claim expectation.

Proposition 1 shows that there exists $(\alpha, M)$ such that the premiums satisfy the expected value principle. We will denote the probability space as a triple 
$(\Omega, \mathcal{F}, \mathbb{P}), \sum_{i=a}^{b} x_{i}=0$ and $\prod_{i=a}^{b} x_{i}=1$ if $a>b$.

Proposition 1. Assuming that

$$
\mathbb{E}\left(X_{1}\right)<\mathbb{E}\left(Y_{1}\right)
$$

for any given $M$ there exists $\alpha$ such that:

$$
\mathbb{E}\left(\min \left\{X_{1}, M\right\}\right)<\alpha \mathbb{E}\left(Y_{1}\right)
$$

and

$$
\mathbb{E}\left(\max \left\{X_{1}-M, 0\right\}\right)<(1-\alpha) \mathbb{E}\left(Y_{1}\right)
$$

Proof. For any $M$, we denote $A=\left\{X_{1} \leq M\right\}$ and $\bar{A}=\Omega \backslash A=\left\{X_{1}>M\right\}$. We have

$$
\begin{aligned}
\mathbb{E}\left(\min \left\{X_{1}, M\right\}\right)=\int_{\Omega} \min & \left\{X_{1}, M\right\} \mathrm{d} \mathbb{P}=\int_{A \cup \bar{A}} \min \left\{X_{1}, M\right\} \mathrm{d} \mathbb{P}=\int_{A} X_{1} \mathrm{~d} \mathbb{P}+\int_{\bar{A}} M \mathrm{~d} \mathbb{P} . \\
\mathbb{E}\left(\max \left\{X_{1}-M, 0\right\}\right) & =\int_{\Omega} \max \left\{X_{1}-M, 0\right\} \mathrm{d} \mathbb{P}=\int_{A \cup \bar{A}} \max \left\{X_{1}-M, 0\right\} \mathrm{d} \mathbb{P} \\
& =\int_{\bar{A}}\left(X_{1}-M\right) \mathrm{d} \mathbb{P}=\int_{\bar{A}} X_{1} \mathrm{~d} \mathbb{P}-\int_{\bar{A}} M \mathrm{~d} \mathbb{P} .
\end{aligned}
$$

Using (2.4) and (2.5), we imply that

$$
\mathbb{E}\left(\min \left\{X_{1}, M\right\}\right)+\mathbb{E}\left(\max \left\{X_{1}-M, 0\right\}\right)=\mathbb{E}\left(X_{1}\right) .
$$

From $\mathbb{E}\left(X_{1}\right)<\mathbb{E}\left(Y_{1}\right)$, thus

$$
\mathbb{E}\left(\min \left\{X_{1}, M\right\}\right)+\mathbb{E}\left(\max \left\{X_{1}-M, 0\right\}\right)<\mathbb{E}\left(Y_{1}\right) .
$$

We have

$$
\frac{\mathbb{E}\left(\min \left\{X_{1}, M\right\}\right)}{\mathbb{E}\left(Y_{1}\right)}<1-\frac{\mathbb{E}\left(\max \left\{X_{1}-M, 0\right\}\right)}{\mathbb{E}\left(Y_{1}\right)} .
$$

Since, we imply the existence $\alpha$ such that

$$
\alpha \in\left(\frac{\mathbb{E}\left(\min \left\{X_{1}, M\right\}\right)}{\mathbb{E}\left(Y_{1}\right)}, 1-\frac{\mathbb{E}\left(\max \left\{X_{1}-M, 0\right\}\right)}{\mathbb{E}\left(Y_{1}\right)}\right)
$$

where $\frac{\mathbb{E}\left(\min \left\{X_{1}, M\right\}\right)}{\mathbb{E}\left(Y_{1}\right)} \geq 0$ and $1-\frac{\mathbb{E}\left(\max \left\{X_{1}-M, 0\right\}\right)}{\mathbb{E}\left(Y_{1}\right)} \leq 1$.

We now consider the surplus process $\left\{U_{n}\right\}_{n>0}$ defined by (1.1) with the excess of loss reinsurance. Then, the cedent's surplus and the reinsurer's surplus in period $n, n=1,2, \cdots$ are denoted by $U_{n}$ and $V_{n}$, respectively. Surpluses $U_{n}$ and $V_{n}$ can be expressed as

$$
U_{n}=u+\alpha \sum_{i=1}^{n} Y_{i}-\sum_{i=1}^{n} \min \left\{X_{i}, M\right\}
$$

and

$$
V_{n}=v+(1-\alpha) \sum_{i=1}^{n} Y_{i}-\sum_{i=1}^{n} \max \left\{X_{i}-M, 0\right\}
$$

where $u$ and $v$ are initial surpluses of the cedent and the reinsurer. The processes 
(2.7) and (2.8) are called surplus processes.

The finite-time and ultimate ruin probability of the cedent with initial surplus $u$ are respectively defined by

$$
\psi_{n}^{(1)}(u, \alpha, M)=\mathbb{P}\left(\bigcup_{i=1}^{n}\left(U_{i} \leq 0\right)\right) \text { and } \psi^{(1)}(u, \alpha, M)=\mathbb{P}\left(\bigcup_{i=1}^{\infty}\left(U_{i} \leq 0\right)\right) .
$$

Similarly, the finite-time and ultimate ruin probability of the reinsurer with initial surplus $v$ are denoted by $\psi_{n}^{(2)}(v, \alpha, M)$ and $\psi^{(2)}(v, \alpha, M)$. The probabilities are defined by

$$
\psi_{n}^{(2)}(v, \alpha, M)=\mathbb{P}\left(\bigcup_{i=1}^{n}\left(V_{i} \leq 0\right)\right) \text { and } \psi^{(2)}(v, \alpha, M)=\mathbb{P}\left(\bigcup_{i=1}^{\infty}\left(V_{i} \leq 0\right)\right) .
$$

Obviously: $\psi^{(1)}(u, \alpha, M)=\lim _{n \rightarrow \infty} \psi_{n}^{(1)}(u, \alpha, M)$ and $\psi^{(2)}(v, \alpha, M)=\lim _{n \rightarrow \infty} \psi_{n}^{(2)}(v, \alpha, M)$.

Let $I^{(1)}=\left\{I_{n}^{(1)}\right\}_{n \geq 0}$ and $I^{(2)}=\left\{I_{n}^{(2)}\right\}_{n \geq 0}$ be the interest rate sequences of the cedent and the reinsurer, respectively. The interest rates satisfy assumptions (2.1) and (2.2).

- Assumption 2.1. The cedent's interest rate sequence $I^{(1)}=\left\{I_{n}^{(1)}\right\}_{n \geq 0}$ is a homogeneous Markov chain, $I_{n}^{(1)}$ takes the values in a finite set of positive $E=\left\{i_{0}, i_{1}, \cdots, i_{M_{1}}\right\}$ and

$$
p_{s t}=\mathbb{P}\left(I_{n+1}^{(1)}=i_{t} \mid I_{n}^{(1)}=i_{s}, I_{n-1}^{(1)}=i_{t_{n-1}}, \cdots, I_{0}^{(1)}=i_{t_{0}}\right)=\mathbb{P}\left(I_{n+1}^{(1)}=i_{t} \mid I_{n}^{(1)}=i_{s}\right)
$$

where $\sum_{t=0}^{M_{1}} p_{s t}=1$ for any $s=0,1, \cdots, M_{1}$ and $p_{s t}>0$ for all $s, t=0,1, \cdots, M_{1}$.

- Assumption 2.2. The reinsurer's interest rate sequence $I^{(2)}=\left\{I_{n}^{(2)}\right\}_{n \geq 0}$ is a homogeneous Markov chain, $I_{n}^{(2)}$ takes the values in a finite set of positive $F=\left\{j_{0}, j_{1}, \cdots, j_{M_{2}}\right\}$ and$$
q_{s t}=\mathbb{P}\left(I_{n+1}^{(2)}=j_{t} \mid I_{n}^{(2)}=j_{s}, I_{n-1}^{(2)}=j_{t_{n-1}}, \cdots, I_{0}^{(2)}=j_{t_{0}}\right)=\mathbb{P}\left(I_{n+1}^{(2)}=j_{t} \mid I_{n}^{(2)}=j_{s}\right)
$$

where $\sum_{t=0}^{M_{2}} q_{s t}=1$ for any $s=0,1, \cdots, M_{2}$ and $q_{s t}>0$ for all $s, t=0,1, \cdots, M_{2}$.

Then, the cedent's surplus and the reinsurer's surplus in period $n, n=1,2, \cdots$ are denoted by $U_{n}^{*}$ and $V_{n}^{*}$.

$$
U_{n}^{*}=U_{n-1}^{*}\left(1+I_{n}^{(1)}\right)+\alpha Y_{n}-\min \left\{X_{n}, M\right\}
$$

and

$$
V_{n}^{*}=V_{n-1}^{*}\left(1+I_{n}^{(2)}\right)+(1-\alpha) Y_{n}-\max \left\{X_{n}-M, 0\right\}
$$

where $U_{0}^{*}=u$ and $V_{0}^{*}=v$ are the cedent' initial surplus and the reinsurer's initial surplus, respectively.

It is easy to see that (2.13) and (2.14) are equivalent to

$$
\begin{aligned}
U_{n}^{*} & =u \prod_{k=1}^{n}\left(1+I_{k}^{(1)}\right)+\sum_{k=1}^{n-1}\left(\alpha Y_{k}-\min \left\{X_{k}, M\right\}\right) \prod_{j=k+1}^{n}\left(1+I_{j}^{(1)}\right)+\alpha Y_{n}-\min \left\{X_{n}, M\right\} \\
& =\prod_{k=1}^{n}\left(1+I_{k}^{(1)}\right)\left[u+\sum_{k=1}^{n}\left(\alpha Y_{k}-\min \left\{X_{k}, M\right\}\right) \prod_{j=1}^{k}\left(1+I_{j}^{(1)}\right)^{-1}\right]
\end{aligned}
$$


and

$$
\begin{aligned}
V_{n}^{*}= & v \prod_{k=1}^{n}\left(1+I_{k}^{(2)}\right)+\sum_{k=1}^{n-1}\left((1-\alpha) Y_{k}-\max \left\{X_{k}-M, 0\right\}\right) \prod_{j=k+1}^{n}\left(1+I_{j}^{(2)}\right) \\
& +(1-\alpha) Y_{n}-\max \left\{X_{n}-M, 0\right\} \\
= & \prod_{k=1}^{n}\left(1+I_{k}^{(2)}\right)\left[v+\sum_{k=1}^{n}\left((1-\alpha) Y_{k}-\max \left\{X_{k}-M, 0\right\}\right) \prod_{j=1}^{k}\left(1+I_{j}^{(2)}\right)^{-1}\right]
\end{aligned}
$$

The finite-time and ultimate the cedent's ruin probabilities with surplus process (2.15), initial surplus $u$ and a given $I_{0}^{(1)}=i_{s}$ are respectively defined by

$$
\phi_{n}^{(1)}\left(u, \alpha, M, i_{s}\right)=\mathbb{P}\left(\bigcup_{k=1}^{n}\left(U_{k}^{*} \leq 0\right) \mid I_{0}^{(1)}=i_{s}\right)
$$

and

$$
\phi^{(1)}\left(u, \alpha, M, i_{s}\right)=\mathbb{P}\left(\bigcup_{k=1}^{\infty}\left(U_{k}^{*} \leq 0\right) \mid I_{0}^{(1)}=i_{s}\right) .
$$

Similarly, the finite-time and ultimate the reinsurer's ruin probabilities with surplus process (2.16), initial surplus $v$ and a given $I_{0}^{(2)}=j_{t}$ are

$$
\phi_{n}^{(2)}\left(v, \alpha, M, j_{t}\right)=\mathbb{P}\left(\bigcup_{k=1}^{n}\left(V_{k}^{*} \leq 0\right) \mid I_{0}^{(2)}=j_{t}\right)
$$

and

$$
\phi^{(2)}\left(v, \alpha, M, j_{t}\right)=\mathbb{P}\left(\bigcup_{k=1}^{\infty}\left(V_{k}^{*} \leq 0\right) \mid I_{0}^{(2)}=j_{t}\right) .
$$

Clearly: $\phi^{(1)}\left(u, \alpha, M, i_{s}\right)=\lim _{n \rightarrow \infty} \phi_{n}^{(1)}\left(u, \alpha, M, i_{s}\right)$ and $\phi^{(2)}\left(v, \alpha, M, j_{t}\right)=\lim _{n \rightarrow \infty} \phi_{n}^{(2)}\left(v, \alpha, M, j_{t}\right)$.

\section{The Ruin Probabilities in the Risk Model without Interest Rate}

The adjustment coefficients, which depend on quota share level and retention level, are established in the following lemmas.

Lemma 2. If essup $\left\{X_{1}\right\}<+\infty$, essup $\left\{Y_{1}\right\}<+\infty, \alpha \mathbb{E}\left(Y_{1}\right)>\mathbb{E}\left(\min \left\{X_{1}, M\right\}\right)$ and $\mathbb{P}\left(\min \left\{X_{1}, M\right\}-\alpha Y_{1}>0\right)>0$ for any $(\alpha, M)$ then there exists the unique $\hat{R}_{0}(\alpha, M)\left(\hat{R}_{0}(\alpha, M)>0\right)$ such that

$$
\mathbb{E}\left(\mathrm{e}^{\hat{R}_{0}(\alpha, M)\left(\min \left\{X_{1}, M\right\}-\alpha Y_{1}\right)}\right)=1 .
$$

Proof. We set $N_{1}=\operatorname{essup}\left(X_{1}\right), N_{2}=\operatorname{essup}\left(Y_{1}\right)$ and $Q_{1}=\min \left\{X_{1}, M\right\}-\alpha Y_{1}$. We have

$$
\left|\mathrm{e}^{R\left(\min \left\{X_{1}, M\right\}-\alpha Y_{1}\right)}\right| \leq \mathrm{e}^{R N_{1}} \text { for all } 0 \leq R<\infty ; \mathbb{E}\left(\mathrm{e}^{R N_{1}}\right)<\infty .
$$

Therefore, there exists the expectation value of $\mathrm{e}^{R\left(\min \left\{X_{1}, M\right\}-\alpha Y_{1}\right)}$ for all $0 \leq R<\infty$.

For any $(\alpha, M)$, if we set $g_{\alpha, M}(R)=\mathbb{E}\left(\mathrm{e}^{R Q_{1}}\right)-1$ for $0 \leq R<\infty$ then 


$$
g_{\alpha, M}(0)=0 .
$$

Differentiating the above function, we get

$$
g_{\alpha, M}^{\prime}(R)=\lim _{\Delta R \rightarrow 0} \frac{g_{\alpha, M}(R+\Delta R)-g_{\alpha, M}(R)}{\Delta R}=\lim _{\Delta R \rightarrow 0} \int_{\Omega} \frac{\mathrm{e}^{R Q_{1}}\left(\mathrm{e}^{\Delta R Q_{1}}-1\right)}{\Delta R} \mathrm{~d} P .
$$

Let $\left\{\Delta R_{n}\right\}_{n \geq 1}\left(\Delta R_{n} \neq 0, R+\Delta R_{n}>0\right), \Delta R_{n} \rightarrow 0$ be an arbitrary real-valued sequence as $n \rightarrow \infty$. Using the Mean Value Theorem for $\mathrm{e}^{t \Delta R_{n} Q_{1}}, t \in[0,1]$, we obtain

$$
\mathrm{e}^{\Delta R_{n} Q_{1}}=1+\Delta R_{n} Q_{1} \mathrm{e}^{\theta \Delta R_{n} Q_{1}},(0<\theta<1)
$$

Moreover, for any $\epsilon>0$, there exists a natural number $n_{0}$ such that $\left|\Delta R_{n}\right|<N_{3}$ for all $n \geq 1$, where $N_{3}=\max \left\{\epsilon,\left|\Delta R_{1}\right|,\left|\Delta R_{2}\right|, \cdots,\left|\Delta R_{n_{0}}\right|\right\}$.

Thus, we have

$$
\left|\frac{\mathrm{e}^{R Q_{1}}\left(\mathrm{e}^{\Delta R_{n} Q_{1}}-1\right)}{\Delta R_{n}}\right|=\left|Q_{1} \mathrm{e}^{\left(R+\theta \Delta R_{n}\right) Q_{1}}\right| \leq\left|Q_{1} \mathrm{e}^{\left(R+\theta N_{3}\right) \mid Q_{1}}\right| \leq\left(N_{1}+N_{2}\right) \mathrm{e}^{\left(R+\theta N_{3}\right)\left(N_{1}+N_{2}\right)}, n \geq 1
$$

and

$$
E\left(\left(N_{1}+N_{2}\right) \mathrm{e}^{\left(R+\theta N_{3}\right)\left(N_{1}+N_{2}\right)}\right)=\left(N_{1}+N_{2}\right) \mathrm{e}^{\left(R+\theta N_{3}\right)\left(N_{1}+N_{2}\right)}<+\infty .
$$

Applying Lebesgue's Dominated Convergence Theorem, we imply that

$\lim _{n \rightarrow \infty} \int_{\Omega} \frac{\mathrm{e}^{R Q_{1}}\left(\mathrm{e}^{\Delta R_{n} Q_{1}}-1\right)}{\Delta R_{n}} \mathrm{~d} P=\int_{\Omega} \lim _{n \rightarrow \infty} \frac{\mathrm{e}^{R Q_{1}}\left(\mathrm{e}^{\Delta R_{n} Q_{1}}-1\right)}{\Delta R_{n}} \mathrm{~d} P=\int_{\Omega} Q_{1} \mathrm{e}^{R Q_{1}} \mathrm{~d} P=\mathbb{E}\left(Q_{1} \mathrm{e}^{R Q_{1}}\right)$.

From (3.3) and (3.4) function $g_{\alpha, M}(R)$ is differentiable

$$
g_{\alpha, M}^{\prime}(R)=\mathbb{E}\left(Q_{1} \mathrm{e}^{R Q_{1}}\right)=\mathbb{E}\left(\left(\min \left\{X_{1}, M\right\}-\alpha Y_{1}\right) \mathrm{e}^{R\left(\min \left\{X_{1}, M\right\}-\alpha Y_{1}\right)}\right) .
$$

So,

$$
g_{\alpha, M}^{\prime}(0)=\mathbb{E}\left(\min \left\{X_{1}, M\right\}-\alpha Y_{1}\right)<0 .
$$

It means that the $g_{\alpha, M}(R)$ is decreasing at $R=0$.

Since $\mathbb{P}\left(\min \left\{X_{1}, M\right\}-\alpha Y_{1}>0\right)>0$ there exists $\delta>0$ so that $\mathbb{P}\left(\min \left\{X_{1}, M\right\}-\alpha Y_{1}>\delta\right)>0$. We have

$$
\begin{aligned}
g_{\alpha, M}(R) & =\mathbb{E}\left(\mathrm{e}^{R\left(\min \left\{X_{1}, M\right\}-\alpha Y_{1}\right)}\right)-1 \\
& \geq \mathbb{E}\left(\mathrm{e}^{R\left(\min \left\{X_{1}, M\right\}-\alpha Y_{1}\right)} 1_{\left(\min \left\{X_{1}, M\right\}-\alpha Y_{1}>\delta\right)}\right)-1 \\
& >\mathbb{E}\left(\mathrm{e}^{R \delta} 1_{\left(\min \left\{X_{1}, M\right\}-\alpha Y_{1}>\delta\right)}\right)-1 \\
& =\mathrm{e}^{R \delta} \mathbb{P}\left(\min \left\{X_{1}, M\right\}-\alpha Y_{1}>\delta\right)-1 .
\end{aligned}
$$

The right side of (3.6) tends to infinity as $R \rightarrow \infty$. It implies that

$$
\lim _{R \rightarrow \infty} g_{\alpha, M}(R)=\infty \text {. }
$$

Combining (3.2), (3.5) and (3.7), function $g_{\alpha, M}(R)$ must intersect the x-axis. In other words, there exists a positive x-intercept of $g_{\alpha, M}(R)$. Let's denote it $\hat{R}_{0}(\alpha, M)\left(\hat{R}_{0}(\alpha, M)>0\right)$. Apparently, $\hat{R}_{0}(\alpha, M)$ is a root of the following 
equation

$$
g_{\alpha, M}(R)=0 .
$$

Similarly, function $g_{\alpha, M}(R)$ is twice differentiable. Hence,

$$
g_{\alpha, M}^{\prime \prime}(R)=\mathbb{E}\left(\left(\min \left\{X_{1}, M\right\}-\alpha Y_{1}\right)^{2} \mathrm{e}^{R\left(\min \left\{X_{1}, M\right\}-\alpha Y_{1}\right)}\right) ; g_{\alpha, M}^{\prime \prime}(R)>0 .
$$

That means $g_{\alpha, M}(R)$ is strictly convex for $R \in[0,+\infty)$. Thus, $\hat{R}_{0}(\alpha, M)$ is the unique positive of Equation (3.8)

The proof of Lemma 3 is similar to Lemma 2 and we omit the proof here.

Lemma 3. If

$$
\operatorname{essup}\left\{X_{1}\right\}<\infty, \operatorname{essup}\left\{Y_{1}\right\}<\infty,(1-\alpha) \mathbb{E}\left(Y_{1}\right)>\mathbb{E}\left(\max \left\{X_{1}-M, 0\right\}\right)
$$

and

$$
\mathbb{P}\left(\max \left\{X_{1}-M, 0\right\}-(1-\alpha) Y_{1}>0\right)>0
$$

for any $(\alpha, M)$ then there exists the unique $\tilde{R}_{0}(\alpha, M)\left(\tilde{R}_{0}(\alpha, M)>0\right)$ such that

$$
\mathbb{E}\left(\mathrm{e}^{\tilde{R}_{0}(\alpha, M)\left(\max \left\{X_{1}-M, 0\right\}-(1-\alpha) Y_{1}\right)}\right)=1 .
$$

The following theorem provides the exponential upper bounds of $\psi^{(1)}(u, \alpha, M)$ and $\psi^{(2)}(v, \alpha, M)$.

Theorem 4. Assuming that the surplus processes given in (2.7) and (2.8) satisfy assumptions in Lemma 2 and Lemma 3. Then,

$$
\psi^{(1)}(u, \alpha, M) \leq \mathrm{e}^{-u \hat{R}_{0}(\alpha, M)}
$$

and

$$
\psi^{(2)}(v, \alpha, M) \leq \mathrm{e}^{-v \tilde{R}_{0}(\alpha, M)}
$$

for any $(\alpha, M)$.

Proof. In order to prove (3.10), we set the stochastic process $\left\{Z_{n}\right\}_{n \geq 0}$ :

$$
Z_{0}=\mathrm{e}^{-u \hat{R}_{0}(\alpha, M)}, Z_{n}=\mathrm{e}^{-\hat{R}_{0}(\alpha, M)\left(u+\sum_{i=1}^{n}\left(\alpha Y_{i}-\min \left\{X_{i}, M\right\}\right)\right)}=\mathrm{e}^{-\hat{R}_{0}(\alpha, M) U_{n}} \text { for } n=1,2, \cdots \quad \text { and }
$$

the filtration $\left\{\mathcal{F}_{n}\right\}_{n \geq 1}$ where $\mathcal{F}_{0}=\{\varnothing, \Omega\}$, $\mathcal{F}_{n}=\sigma\left(Z_{1}, Z_{2}, \cdots, Z_{n}\right)=\sigma\left(X_{1}, X_{2}, \cdots, X_{n}, Y_{1}, Y_{2}, \cdots, Y_{n}\right) ; n=1,2, \cdots$

The stochastic process $\left\{Z_{n}\right\}_{n \geq 0}$ is a martingale with respect to the filtration $\left\{\mathcal{F}_{n}\right\}_{n \geq 0}$.

Indeed

$$
\begin{aligned}
E\left(Z_{1} \mid \mathcal{F}_{0}\right) & =E\left(\mathrm{e}^{-\hat{R}_{0}(\alpha, M)\left(u+\alpha Y_{1}-\min \left\{X_{1}, M\right\}\right)}\right) \\
& =E\left(\mathrm{e}^{-u \hat{R}_{0}(\alpha, M)} \mathrm{e}^{\hat{R}_{0}(\alpha, M)\left(\min \left\{X_{1}, M\right\}-\alpha Y_{1}\right)}\right) \\
& =\mathrm{e}^{-u \hat{R}_{0}(\alpha, M)}=Z_{0} .
\end{aligned}
$$

We now consider for $n=1,2, \cdots$ 


$$
\begin{aligned}
& \mathbb{E}\left(Z_{n+1} \mid \mathcal{F}_{n}\right) \\
& =\mathbb{E}\left(Z_{n} \mathrm{e}^{-\hat{R}_{0}(\alpha, M)\left(\alpha Y_{n+1}-\min \left\{X_{n+1}, M\right\}\right)} \mid \mathcal{F}_{n}\right) \\
& =Z_{n} \mathbb{E}\left(\mathrm{e}^{\hat{R}_{0}(\alpha, M)\left(\min \left\{X_{n+1}, M\right\}-\alpha Y_{n+1}\right)} \mid \mathcal{F}_{n}\right) \\
& =Z_{n} \mathbb{E}\left(\mathrm{e}^{\hat{R}_{0}(\alpha, M)\left(\min \left\{X_{n+1}, M\right\}-\alpha Y_{n+1}\right)} \mid X_{1}, X_{2}, \cdots, X_{n}, Y_{1}, Y_{2}, \cdots, Y_{n}\right) \\
& =Z_{n} \mathbb{E}\left(\mathrm{e}^{\hat{R}_{0}(\alpha, M)\left(\min \left\{X_{n+1}, M\right\}-\alpha Y_{n+1}\right)}\right) .
\end{aligned}
$$

Moreover

$$
\mathbb{E}\left(\mathrm{e}^{\hat{R}_{0}(\alpha, M)\left(\min \left\{X_{n+1}, M\right\}-\alpha Y_{n+1}\right)}\right)=\mathbb{E}\left(\mathrm{e}^{\hat{R}_{0}(\alpha, M)\left(\min \left\{X_{1}, M\right\}-\alpha Y_{1}\right)}\right)=1 .
$$

Combining (3.13) and (3.14), thus

$$
\mathbb{E}\left(Z_{n+1} \mid \mathcal{F}_{n}\right)=Z_{n} \text { for } n=1,2, \cdots
$$

Since the stochastic process $\left\{Z_{n}\right\}_{n \geq 0}$ is a martingale with respect to the filtration $\left\{\mathcal{F}_{n}\right\}_{n \geq 0}$. Let $\tau=\min \left\{n: U_{n} \leq 0\right\}$. Then $n \wedge \tau=\min (n, \tau)$ is a finite stopping time. Thus, using the optional stopping theorem for martingale $\left\{Z_{n}\right\}_{n \geq 0}$, (see [10]) we get

$$
\mathbb{E}\left(Z_{n \wedge \tau}\right)=\mathbb{E}\left(Z_{0}\right)=\mathrm{e}^{-u \hat{R}_{0}(\alpha, M)} .
$$

This deduces that

$$
\mathrm{e}^{-u \hat{R}_{0}(\alpha, M)}=\mathbb{E}\left(Z_{n \wedge \tau}\right) \geq \mathbb{E}\left(Z_{n \wedge \tau} 1_{(\tau \leq n)}\right)=\mathbb{E}\left(Z_{\tau} 1_{(\tau \leq n)}\right) .
$$

From (3.16) and $\left(Z_{\tau} \geq 1\right)$, we obtain

$$
\mathrm{e}^{-u \hat{k}_{0}(\alpha, M)} \geq \mathbb{E}\left(1_{\tau \leq n}\right)=\mathbb{P}(\tau \leq n)=\psi_{n}^{(1)}(u, \alpha, M) .
$$

Therefore, inequality (3.10) is followed by letting $n \rightarrow \infty$ in (3.17).

The proof of inequality (3.11) is similar to the one for inequality (3.10).

In reinsurance businesses, evaluation the two ruin probabilities of the cedent and the reinsurer are crucial. Because the insurers based on the ruin probabilities to determine $(\alpha, M)$ so that $\psi^{(1)}(u, \alpha, M)$ and $\psi^{(2)}(v, \alpha, M)$ are decreased. However, the issue is a difficult topic. The following theorem shows us how to determine $(\alpha, M)$ so that $\psi_{1}^{(1)}(u, \alpha, M)$ and $\psi_{1}^{(2)}(v, \alpha, M)$ are less than a given value $\epsilon$.

Theorem 5. Assuming that the surplus processes given in (2.7) and (2.8) satisfy the following assumptions:

1) Random variable $X_{n}, n=1,2, \cdots$ takes values in a finite set of non-negative numbers $G_{X}=\left\{x_{1}, x_{2}, \cdots, x_{N}\right\}$ where $\left(0 \leq x_{1}<x_{2}<\cdots<x_{N}\right)$ and $p_{k}=\mathbb{P}\left(X_{n}=x_{k}\right), \quad 0<p_{k} \leq 1, \quad \sum_{k=1}^{N} p_{k}=1$;

2) $x_{N}-u-v>0$.

For any given $\epsilon$ satisfies

$$
P\left(Y_{1} \leq x_{N}-u-v\right) P\left(\bigcup_{i=1, N: x_{i} \geq u}\left(X_{1}=x_{i}\right)\right) \leq \epsilon
$$


there exists $(\alpha, M)$ such that

$$
\psi_{1}^{(1)}(u, \alpha, M) \leq \epsilon
$$

and

$$
\psi_{1}^{(2)}(v, \alpha, M) \leq \epsilon
$$

Proof. By $x_{N}-u-v>0$, we imply that there exists $(M, \alpha)$ such that

$$
M=u+\alpha\left(x_{N}-u-v\right) \text { for all } \alpha \in(0,1) .
$$

Obviously $M>u$.

Expression (3.21) is equivalent to

$$
\frac{M-u}{\alpha}=x_{N}-u-v .
$$

For any given $\epsilon$ satisfies (3.18). We have

$$
\begin{aligned}
& \epsilon \geq \mathbb{P}\left(Y_{1} \leq x_{N}-u-v\right) \mathbb{P}\left(\underset{i=1, N: x_{i} \geq u}{\bigcup_{i}}\left(X_{1}=x_{i}\right)\right) \\
& =\mathbb{P}\left(Y_{1} \leq \frac{M-u}{\alpha}\right) \mathbb{P}\left(\underset{i=1, N: x_{i} \geq u}{\bigcup_{i}}\left(X_{1}=x_{i}\right)\right) \\
& =\mathbb{P}\left(Y_{1} \leq \frac{M-u}{\alpha}\right) \mathbb{P}\left(\left[\bigcup_{i=1, N: M \geq x_{i} \geq u}\left(X_{1}=x_{i}\right)\right] \cup\left[\underset{i=1, N: x_{i}>M}{\bigcup_{1}}\left(X_{1}=x_{i}\right)\right]\right) \\
& =\mathbb{P}\left(Y_{1} \leq \frac{M-u}{\alpha}\right) \mathbb{P}\left(\bigcup_{i=1, N: M \geq x_{i} \geq u}\left(X_{1}=x_{i}\right)\right) \\
& +\mathbb{P}\left(Y_{1} \leq \frac{M-u}{\alpha}\right) \mathbb{P}\left(\bigcup_{i=1, N: x_{i}>M}\left(X_{1}=x_{i}\right)\right) \\
& =\mathbb{P}\left(\left[Y_{1} \leq \frac{M-u}{\alpha}\right] \cap\left[\bigcup_{i=1, N: M \geq x_{i} \geq u}\left(X_{1}=X_{i}\right)\right]\right) \\
& +\mathbb{P}\left(\left[Y_{1} \leq \frac{M-u}{\alpha}\right] \cap\left[\bigcup_{i=1, N: x_{i}>M}\left(X_{1}=x_{i}\right)\right]\right) \\
& \geq \mathbb{P}\left(\left[Y_{1} \leq \frac{X_{1}-u}{\alpha}\right] \cap\left[\bigcup_{i=1, N: M \geq x_{i} \geq u}\left(X_{1}=x_{i}\right)\right]\right) \\
& +\mathbb{P}\left(\left[Y_{1} \leq \frac{M-u}{\alpha}\right] \cap\left[\underset{i=1, N: x_{i}>M}{\bigcup}\left(X_{1}=x_{i}\right)\right]\right) \\
& =\mathbb{P}\left(\left[u+\alpha Y_{1}-X_{1} \leq 0\right] \cap\left[\bigcup_{i=1, N: M \geq x_{i} \geq u}\left(X_{1}=x_{i}\right)\right]\right) \\
& +\mathbb{P}\left(\left[u+\alpha Y_{1}-M \leq 0\right] \cap\left[\bigcup_{i=1, N: x_{i}>M}\left(X_{1}=x_{i}\right)\right]\right) \\
& =\mathbb{P}\left(\left[u+\alpha Y_{1}-\min \left\{X_{1}, M\right\} \leq 0\right] \cap\left[\bigcup_{i=1, N: x_{i} \geq u}\left(X_{1}=x_{i}\right)\right]\right) \\
& =\mathbb{P}\left(u+\alpha Y_{1}-\min \left\{X_{1}, M\right\} \leq 0\right)=\psi_{1}^{(1)}(u, \alpha, M) \text {. }
\end{aligned}
$$


Since, $\psi_{1}^{(1)}(u, \alpha, M) \leq \epsilon$.

Moreover, Expression (3.21) can be written

$$
\frac{x_{N}-M-v}{1-\alpha}=x_{N}-u-v
$$

Hence

$$
\begin{aligned}
\epsilon & \geq \mathbb{P}\left(Y_{1} \leq x_{N}-u-v\right) \mathbb{P}\left(\bigcup_{i=1, N: x_{i} \geq u}\left(X_{1}=x_{i}\right)\right) \\
& =\mathbb{P}\left(Y_{1} \leq \frac{X_{N}-M-v}{1-\alpha}\right) \mathbb{P}\left(\bigcup_{i=1, N: x_{i} \geq u}\left(X_{1}=x_{i}\right)\right)
\end{aligned}
$$

Using (3.22) and $M+v>u$, thus

$$
\begin{aligned}
\epsilon & \geq \mathbb{P}\left(Y_{1} \leq \frac{x_{N}-M-v}{1-\alpha}\right) \mathbb{P}\left(\bigcup_{i=1, N: x_{i} \geq M+v}\left(X_{1}=x_{i}\right)\right) \\
& =\mathbb{P}\left(\left[Y_{1} \leq \frac{X_{N}-M-v}{1-\alpha}\right] \cap\left[\bigcup_{i=1, N: x_{i} \geq M+v}\left(X_{1}=x_{i}\right)\right]\right) \\
& \geq \mathbb{P}\left(\left[Y_{1} \leq \frac{X_{1}-M-v}{1-\alpha}\right] \cap\left[\bigcup_{i=1, N: x_{i} \geq M+v}\left(X_{1}=x_{i}\right)\right]\right) \\
& =\mathbb{P}\left(\left[v+(1-\alpha) Y_{1}-\left(X_{1}-M\right) \leq 0\right] \cap\left[\bigcup_{i=1, N: x_{i} \geq M+v}\left(X_{1}=x_{i}\right)\right]\right) \\
& \left.=\mathbb{P}\left(\left[v+(1-\alpha) Y_{1}-\max \left\{X_{1}-M, 0\right\} \leq 0\right] \cap \bigcup_{i=1, N: x_{i} \geq M+v}\left(X_{1}=x_{i}\right)\right]\right) \\
& =\mathbb{P}\left(v+(1-\alpha) Y_{1}-\max \left\{X_{1}-M, 0\right\} \leq 0\right) \\
& =\psi_{1}^{(2)}(v, \alpha, M) .
\end{aligned}
$$

Li [9] investigated the optimal $M$ to maximize the joint survival probability for the cedent and the reinsurer in one period insurance. If both companies don't occur ruin at certain period $n-1, U_{n-1}$ and $V_{n-1}$ will be initial surpluses of the insurance companies before period $n$, respectively. Therefore, we apply Theorem 5 to estimate the probabilities of the insurance companies to period $n-1$ from period $n$.

\section{The Ruin Probabilities in the Risk Model with Interest Rate}

In the section, we consider surplus processes (2.15) and (2.16). The proofs of Lemma 6 and Lemma 7 are similar to the one for Lemma 2.

Lemma 6. If

$\operatorname{essup}\left\{X_{1}\right\}<+\infty, \operatorname{essup}\left\{Y_{1}\right\}<+\infty, \alpha \mathbb{E}\left(Y_{1}\right)>\mathbb{E}\left(\min \left\{X_{1}, M\right\}\right)$ and $\mathbb{P}\left(\min \left\{X_{1}, M\right\}-\alpha Y_{1}>0\right)>0$ for any $(\alpha, M)$ then there exists the unique $\hat{R}_{i_{s}}(\alpha, M)\left(\hat{R}_{i_{s}}(\alpha, M)>0\right)$ such that 


$$
\mathbb{E}\left(\mathrm{e}^{\hat{R}_{i_{s}}(\alpha, M)\left(\min \left\{X_{1}, M\right\}-\alpha Y_{1}\right)\left(1+I_{1}^{(1)}\right)^{-1}} \mid I_{0}^{(1)}=i_{s}\right)=1
$$

for any $s, s=0,1, \cdots, M_{1}$.

Proof. For any $(\alpha, M)$ and $s, s=0,1, \cdots, M_{1}$, we set

$$
f_{\alpha, M, s}(R)=\mathbb{E}\left(\mathrm{e}^{R\left(\min \left\{X_{1}, M\right\}-\alpha Y_{1}\right)\left(1+I_{1}^{(1)}\right)^{-1}} \mid I_{0}^{(1)}=i_{s}\right)-1
$$

for $R \in[0, \infty)$.

Similarly, we show the expectation value existence in (4.1). In particular

$$
f_{\alpha, M, s}(0)=0 \text {, }
$$

$$
\begin{aligned}
f_{\alpha, M, s}^{\prime}(0) & =\mathbb{E}\left(\left(\min \left\{X_{1}, M\right\}-\alpha Y_{1}\right)\left(1+I_{1}^{(1)}\right)^{-1} \mid I_{0}^{(1)}=i_{s}\right) \\
& =\frac{\mathbb{E}\left(\min \left\{X_{1}, M\right\}-\alpha Y_{1}\right)}{\mathbb{E}\left(\left(1+I_{1}^{(1)}\right) \mid I_{0}^{(1)}=i_{s}\right)}<0,
\end{aligned}
$$

$$
f_{\alpha, M, s}^{\prime \prime}(R)=\mathbb{E}\left(\left[\left(\min \left\{X_{1}, M\right\}-\alpha Y_{1}\right)\left(1+I_{1}^{(1)}\right)^{-1}\right]^{2} \mathrm{e}^{R\left(\min \left\{X_{1}, M\right\}-\alpha Y_{1}\right)\left(1+I_{1}^{(1)}\right)^{-1}} \mid I_{0}^{(1)}=i_{s}\right)>0 .
$$

Moreover

$$
\lim _{R \rightarrow+\infty} f_{\alpha, M, s}(R)=+\infty
$$

Combining assertions to (4.3) from (4.6), we deduce that function $f_{\alpha, M, s}(R)$ must intersect the X-axis. Let's denote it $\hat{R}_{i_{s}}(\alpha, M)\left(\hat{R}_{i_{s}}(\alpha, M)>0\right)$. Apparently, $\hat{R}_{i_{s}}(\alpha, M)$ is the unique intersection.

For any $(\alpha, M)$. We set

$$
\hat{R}_{1}(\alpha, M)=\min _{i_{s} \in E}\left\{\hat{R}_{i_{s}}(\alpha, M)>0: f_{\alpha, M, s}\left(\hat{R}_{i_{s}}(\alpha, M)\right)=1\right\} .
$$

The function $f_{\alpha, M, s}(R)$ is strictly convex for $R \in[0, \infty), 0<\hat{R}_{1}(\alpha, M) \leq R_{i_{s}}$. Since, $f_{\alpha, M, s}\left(\hat{R}_{1}(\alpha, M)\right) \leq 0$ this is equivalent to

$$
\mathbb{E}\left(\mathrm{e}^{\hat{R}_{1}(\alpha, M)\left(\min \left\{X_{1}, M\right\}-\alpha Y_{1}\right)\left(1+I_{1}^{(1)}\right)^{-1}} \mid I_{0}^{(1)}=i_{s}\right) \leq 1
$$

for all $s=0,1, \cdots, M_{1}$.

We have

$$
\begin{aligned}
& \mathbb{E}\left(\mathrm{e}^{\hat{R}_{1}(\alpha, M)\left(\min \left\{X_{1}, M\right\}-\alpha Y_{1}\right)\left(1+I_{1}^{(1)}\right)^{-1}} \mid I_{0}^{(1)}\right) \\
& =\sum_{s=0}^{M_{1}} \mathbb{E}\left(\mathrm{e}^{\hat{R}_{1}(\alpha, M)\left(\min \left\{X_{1}, M\right\}-\alpha Y_{1}\right)\left(1+I_{1}^{(1)}\right)^{-1}} \mid I_{0}^{(1)}=i_{s}\right) 1_{\left(I_{0}^{(1)}=i_{s}\right)} \\
& \leq \sum_{s=0}^{M_{1}} 1_{\left(I_{0}^{(1)}=i_{s}\right)}=1 .
\end{aligned}
$$

Hence 


$$
\mathbb{E}\left(\mathrm{e}^{\hat{R}_{1}(\alpha, M)\left(\min \left\{X_{1}, M\right\}-\alpha Y_{1}\right)\left(1+I_{1}^{(1)}\right)^{-1}} \mid I_{0}^{(1)}\right) \leq 1
$$

\section{Lemma 7. If}

$$
\operatorname{essup}\left\{X_{1}\right\}<+\infty, \operatorname{essup}\left\{Y_{1}\right\}<+\infty,(1-\alpha) \mathbb{E}\left(Y_{1}\right)>\mathbb{E}\left(\max \left\{X_{1}-M, 0\right\}\right)
$$

and

$$
\mathbb{P}\left(\max \left\{X_{1}-M, 0\right\}-(1-\alpha) Y_{1}>0\right)>0
$$

for any $(\alpha, M)$ then there exists the unique $\tilde{R}_{j_{t}}(\alpha, M)\left(\tilde{R}_{j_{t}}(\alpha, M)>0\right)$ such that

$$
\mathbb{E}\left(\mathrm{e}^{\tilde{R}_{j_{t}}(\alpha, M)\left(\max \left\{X_{1}-M, 0\right\}-(1-\alpha) Y_{1}\right)\left(1+I_{1}^{(2)}\right)^{-1}} \mid I_{0}^{(2)}=j_{t}\right)=1
$$

for any $t, t=0,1, \cdots, M_{2}$.

Proof. The proof of Lemma 7 is similar to the one for Lemma 2.

If

$$
\tilde{R}_{1}(\alpha, M)=\min _{j_{s} \in F}\left\{\tilde{R}_{j_{t}}(\alpha, M)>0: g_{\alpha, M, t}\left(\tilde{R}_{j_{t}}(\alpha, M)\right)=1\right\}
$$

then

$$
\mathbb{E}\left(\mathrm{e}^{\tilde{R}_{1}(\alpha, M)\left(\max \left\{X_{1}-M, 0\right\}-(1-\alpha) Y_{1}\right)\left(1+I_{1}^{(2)}\right)^{-1}} \mid I_{0}^{(2)}\right) \leq 1
$$

where

$$
g_{\alpha, M, t}(R)=\mathbb{E}\left(\mathrm{e}^{R\left(\max \left\{X_{1}-M, 0\right\}-(1-\alpha) Y_{1}\right)\left(1+I_{1}^{(2)}\right)^{-1}} \mid I_{0}^{(2)}=j_{t}\right)
$$

Using martingale method, we present the exponential upper bounds of $\phi^{(1)}\left(u, \alpha, M, i_{s}\right)$ and $\phi^{(2)}\left(v, \alpha, M, j_{t}\right)$.

Theorem 8. Assuming that the surplus processes given in (2.15) and (2.16) satisfy assumptions in Lemma 6 and Lemma 7. For any $(\alpha, M)$ then

$$
\phi^{(1)}\left(u, \alpha, M, i_{s}\right) \leq \mathrm{e}^{-u \hat{R}_{1}(\alpha, M)}
$$

and

$$
\phi^{(2)}\left(v, \alpha, M, j_{t}\right) \leq \mathrm{e}^{-v \tilde{R}_{1}(\alpha, M)}
$$

for all $s=0,1, \cdots, M_{1}$ and $t=0,1, \cdots, M_{2}$.

Proof. We first consider the stochastic process $\left\{H_{n}\right\}_{n \geq 0}$ and the filtration $\left\{\mathcal{G}_{n}\right\}_{n \geq 0}$ where

$$
H_{0}=\mathrm{e}^{-u \hat{R}_{1}(\alpha, M)}, H_{n}=\mathrm{e}^{-\hat{R}_{1}(\alpha, M)\left[u+\sum_{k=1}^{n}\left(\alpha Y_{k}-\min \left\{X_{k}, M\right\}\right) \prod_{j=1}^{k}\left(1+I_{j}^{(1)}\right)^{-1}\right]}
$$

and

$$
\mathcal{G}_{0}=\sigma\left(I_{0}^{(1)}\right), \mathcal{G}_{n}=\sigma\left(X_{1}, X_{2}, \cdots, X_{n}, Y_{1}, Y_{2}, \cdots, Y_{n}, I_{0}^{(1)}, I_{1}^{(1)}, \cdots, I_{n}^{(1)}\right), n=1,2, \cdots
$$

We have 


$$
\begin{aligned}
\mathbb{E}\left(H_{1} \mid \mathcal{G}_{0}\right) & =\mathbb{E}\left(\mathrm{e}^{-u \hat{R}_{1}(\alpha, M)+\hat{R}_{1}(\alpha, M)\left(\min \left\{X_{1}, M\right\}-\alpha Y_{1}\right)\left(1+I_{1}^{(1)}\right)^{-1}} \mid I_{0}^{(1)}\right) \\
& =\mathrm{e}^{-u \hat{R}_{1}(\alpha, M)} \mathbb{E}\left(\mathrm{e}^{\hat{R}_{1}(\alpha, M)\left(\min \left\{X_{1}, M\right\}-\alpha Y_{1}\right)\left(1+I_{1}^{(1)}\right)^{-1}} \mid I_{0}^{(1)}\right) \\
& \leq \mathrm{e}^{-u \hat{R}_{1}(\alpha, M)}=H_{0} .
\end{aligned}
$$

For $n=1,2, \cdots$, we get

$$
\begin{aligned}
& \mathbb{E}\left(H_{n+1} \mid \mathcal{G}_{n}\right) \\
& =H_{n} \mathbb{E}\left(\mathrm{e}^{\hat{R}_{1}(\alpha, M)\left(\min \left\{X_{n+1}, M\right\}-\alpha Y_{n+1}\right) \prod_{j=1}^{n+1}\left(1+I_{j}^{(1)}\right)^{-1}} \mid X_{1}, X_{2}, \cdots, X_{n}, Y_{1}, Y_{2}, \cdots, Y_{n}, I_{0}^{(1)}, I_{1}^{(1)}, \cdots, I_{n}^{(1)}\right) \\
& =H_{n} \mathbb{E}\left(\mathrm{e}^{\hat{R}_{1}(\alpha, M)\left(\min \left\{X_{n+1}, M\right\}-\alpha Y_{n+1}\right) \prod_{j=1}^{n+1}\left(1+I_{j}^{(1)}\right)^{-1}} \mid I_{0}^{(1)}, I_{1}^{(1)}, \cdots, I_{n}^{(1)}\right)
\end{aligned}
$$

We set $r=\prod_{j=1}^{n}\left(1+I_{j}^{(1)}\right)^{-1},(0<r \leq 1)$. According to Jensen's Inequality, it implies that

$$
\begin{aligned}
& {\left[\mathbb{E}\left(\mathrm{e}^{\hat{R}_{1}(\alpha, M)\left(\min \left\{X_{n+1}, M\right\}-\alpha Y_{n+1}\right)\left(1+I_{n+1}^{(1)}\right)^{-1} r} \mid I_{0}^{(1)}, I_{1}^{(1)}, \cdots, I_{n}^{(1)}\right)\right]^{1 / r}} \\
& \leq \mathbb{E}\left(\mathrm{e}^{\hat{R}_{1}(\alpha, M)\left(\min \left\{X_{n+1}, M\right\}-\alpha Y_{n+1}\right)\left(1+I_{n+1}^{(1)}\right)^{-1}} \mid I_{n}^{(1)}\right) \\
& =\mathbb{E}\left(\mathrm{e}^{\hat{R}_{1}(\alpha, M)\left(\min \left\{X_{1}, M\right\}-\alpha Y_{1}\right)\left(1+I_{1}^{(1)}\right)^{-1}} \mid I_{0}^{(1)}\right) \leq 1 .
\end{aligned}
$$

Combining (4.17) and (4.18), we obtain

$$
\mathbb{E}\left(H_{n+1} \mid \mathcal{G}_{n}\right) \leq H_{n} \text { for } n=1,2, \cdots
$$

Hence, the stochastic process $\left\{H_{n}\right\}_{n \geq 0}$ is a supermartingale with respect to the filtration $\left\{\mathcal{G}_{n}\right\}_{n \geq 0}$.

Let $\tau_{s}=\min \left\{n: u+\sum_{k=1}^{n}\left(\alpha Y_{k}-\min \left\{X_{k}, M\right\}\right) \prod_{j=1}^{k}\left(1+I_{j}^{(1)}\right)^{-1} \leq 0 \mid I_{0}^{(1)}=i_{s}\right\}$. Then $n \wedge \tau_{s}=\min \left\{n, \tau_{s}\right\}$ is a finite stopping time. Thus, by the optional stopping theorem for supermartingale $\left\{H_{n}\right\}_{n \geq 0}$, (see [10]), we get

$$
\mathbb{E}\left(H_{n \wedge \tau_{s}}\right) \leq \mathbb{E}\left(H_{0}\right)=\mathrm{e}^{-u \hat{R}_{1}(\alpha, M)} .
$$

This implies that

$$
\mathrm{e}^{-u \hat{R}_{1}(\alpha, M)} \geq \mathbb{E}\left(H_{n \wedge \tau_{s}}\right) \geq \mathbb{E}\left(H_{n \wedge \tau_{s}} 1_{\left(\tau_{s} \leq n\right)}\right)=\mathbb{E}\left(H_{\tau_{s}} 1_{\left(\tau_{s} \leq n\right)}\right) .
$$

By $\left(H_{\tau_{\mathrm{s}}} \geq 1\right)$ and (4.19), we have

$$
\mathrm{e}^{-u \hat{R}_{1}(\alpha, M)} \geq \mathbb{E}\left(1_{\tau_{s} \leq n}\right)=\mathbb{P}\left(\tau_{s} \leq n\right)=\phi_{n}^{(1)}\left(u, \alpha, M, i_{s}\right) .
$$

By letting $n \rightarrow \infty$ in (4.20), we obtain inequality (4.14). 
The proof of inequality (4.15) is similar to the one for inequality (4.14).

\section{Numerical Illustrations}

\subsection{Example 5.1}

Suppose that sequences $Y=\left\{Y_{n}\right\}_{n \geq 1}$ and $X=\left\{X_{n}\right\}_{n \geq 1}$ satisfy the conditions in Theorem 5. Initial surpluses $u=2.1$ and $v=1.5$. The distribution functions of $Y_{1}$ and $X_{1}$ are defined in Table 1 and Table 2, respectively:

Inequality (3.18) implies for all $\epsilon \geq 0.028643$ there exists $(\alpha, M)$ such that $\psi_{1}^{(1)}(u, \alpha, M) \leq \epsilon$ and $\psi_{1}^{(2)}(v, \alpha, M) \leq \epsilon$. E.g. from (3.21) we chose $\alpha=0.758614$ this follows $M=2.403446$ then couple $(\alpha=0.758614, M=2.403446)$ is the solution of Theorem 5 .

If $(\alpha=0.758614, M=2.403446)$ then $\mathbb{E}\left(Y_{1}\right)=2.500000$, $\mathbb{E}\left(\min X_{1}, M\right)=1.274934$ and $\mathbb{E}\left(\max \left\{X_{1}-M, 0\right\}\right)=0.222952$. Hence, couple $(\alpha=0.758614, M=2.403446)$ also satisfy Proposition 1 .

\subsection{Example 5.2}

In this example, let $Y_{1}$ and $X_{1}$ take the same structure and values as the ones in Example 5.1. Initial surpluses $u=12.1$ and $v=10.5$.

The interest rate sequence of the cedent $I^{(1)}=\left\{I_{n}^{(1)}\right\}_{n \geq 0}$ is a homogeneous Markov chain, $I_{n}^{(1)}$ takes values: $i_{0}=6 \%, i_{1}=8 \%$ and $i_{2}=10 \%$. The transition probability matrix of the process $I^{(1)}$ is

$$
\left(\begin{array}{lll}
0.3 & 0.6 & 0.1 \\
0.3 & 0.4 & 0.3 \\
0.2 & 0.7 & 0.1
\end{array}\right) .
$$

Similarly, the interest rate sequence of the reinsurer $I^{(2)}=\left\{I_{n}^{(1)}\right\}_{n \geq 0}$ is a homogeneous Markov chain, $I_{n}^{(1)}$ takes values: $j_{0}=5.5 \%, j_{1}=8 \%$ and $j_{2}=12 \%$. The transition probability matrix of the process $I^{(2)}$ is

$$
\left(\begin{array}{ccc}
0.2 & 0.8 & 0 \\
0.15 & 0.7 & 0.15 \\
0 & 0.8 & 0.2
\end{array}\right) .
$$

Let $\alpha=0.350000$ and $M=1.103245$, we have Table 3 and Table 4 .

We denote the moment-generating functions of $W_{1}=\min \left\{X_{1}, M\right\}-\alpha Y_{1}$ and $W_{2}=\max \left\{X_{1}-M, 0\right\}-(1-\alpha) Y_{1}$ are

Table 1. Distribution function of $Y_{1}$.

\begin{tabular}{cccc}
\hline$Y_{1}$ & 0 & 1 & 5 \\
$P$ & 0.205112 & 0.366128 & 0.42876 \\
\hline
\end{tabular}

Table 2. Distribution function of $X_{1}$.

\begin{tabular}{cccc}
\hline$X_{1}$ & 0 & 2 & 4 \\
$P$ & 0.390703 & 0.469651 & 0.139646 \\
\hline
\end{tabular}


Table 3. Distribution function of $\min \left\{X_{1}, M\right\}-\alpha Y_{1}$.

\begin{tabular}{cccc}
\hline $\min \left\{X_{1}, M\right\}-\alpha Y_{1}$ & -1.750000 & -0.646760 \\
$P$ & & 0.167518 & 0.261242 \\
\hline & & \\
\hline-0.350000 & 0.000000 & 0.753245 & 1.103245 \\
0.143047 & 0.080138 & 0.223081 & 0.124974 \\
\hline
\end{tabular}

Table 4. Distribution function of $\max \left\{X_{1}-M, 0\right\}-(1-\alpha) Y_{1}$.

\begin{tabular}{|c|c|c|c|}
\hline \multicolumn{2}{|c|}{$\max \left\{X_{1}-M, 0\right\}-(1-\alpha) Y_{1}$} & \multicolumn{2}{|c|}{-3.250000} \\
\hline \multicolumn{2}{|c|}{$P$} & \multicolumn{2}{|c|}{0.167518} \\
\hline-2.353250 & -0.650000 & -0.353250 & 0.000000 \\
\hline 0.201368 & 0.143047 & 0.059875 & 0.080138 \\
\hline 0.246755 & 0.896755 & 2.246755 & 2.896755 \\
\hline 0.171952 & 0.096331 & 0.051128 & 0.028643 \\
\hline
\end{tabular}

$$
\begin{aligned}
M_{W_{1}}(R)= & \mathbb{E}\left(\mathrm{e}^{R\left(\min \left\{X_{1}, M\right\}-\alpha Y_{1}\right)}\right) \\
= & 0.167518 \mathrm{e}^{-1.750000 R}+0.261242 \mathrm{e}^{-0.646760 R}+0.143047 \mathrm{e}^{-0.350000 R} \\
& +0.080138+0.223081 \mathrm{e}^{0.753245 R}+0.124974 \mathrm{e}^{1.103245 R}
\end{aligned}
$$

and

$$
\begin{aligned}
M_{W_{2}}(R)= & \mathbb{E}\left(\mathrm{e}^{R\left(\max \left\{X_{1}-M, 0\right\}-(1-\alpha) Y_{1}\right)}\right) \\
= & 0.167518 \mathrm{e}^{-3.250000 R}+0.201368 \mathrm{e}^{-2.353250 R}+0.143047 \mathrm{e}^{-0.650000 R} \\
& +0.059875 \mathrm{e}^{-0.353250 R}+0.080138+0.171952 \mathrm{e}^{0.246755 R} \\
& +0.096331 \mathrm{e}^{0.896755 R}+0.051128 \mathrm{e}^{2.246755 R}+0.028643 \mathrm{e}^{2.896755 R}
\end{aligned}
$$

for $R \in(0, \infty)$.

Using Matlab software, we obtain $\hat{R}_{0}(\alpha=0.035000, M=1.103245)=0.493945$ and $\tilde{R}_{0}(\alpha=0.035000, M=1.103245)=0.571825$ which are the solutions of equations $M_{W_{1}}(R)=1$ and $M_{W_{2}}(R)=1$, respectively.

If $I_{0}^{(1)}=i_{0}$ then Equation (4.1) can be written

$$
0.3 M_{W_{1}}\left(\frac{R}{1.06}\right)+0.6 M_{W_{1}}\left(\frac{R}{1.08}\right)+0.1 M_{W_{1}}\left(\frac{R}{1.1}\right)=1 .
$$

Similarly, for $I_{0}^{(1)}=i_{1}$ and $I_{0}^{(1)}=i_{2}$ Equation (4.1) is equivalent to, respectively,

$$
0.3 M_{W_{1}}\left(\frac{R}{1.06}\right)+0.4 M_{W_{1}}\left(\frac{R}{1.08}\right)+0.3 M_{W_{1}}\left(\frac{R}{1.1}\right)=1
$$

and

$$
0.2 M_{W_{1}}\left(\frac{R}{1.06}\right)+0.7 M_{W_{1}}\left(\frac{R}{1.08}\right)+0.1 M_{W_{1}}\left(\frac{R}{1.1}\right)=1 .
$$


Combining the solutions of Equations (5.3), (5.4) and (5.5), we have $\hat{R}_{1}(\alpha=0.350000, M=1.103245)=0.531355$

Similarly, for $I_{0}^{(2)}=j_{0}, I_{0}^{(2)}=j_{1}$ and $I_{0}^{(2)}=j_{2}$ Equation (4.11) can be written, respectively,

$$
\begin{gathered}
0.2 M_{W_{2}}\left(\frac{R}{1.055}\right)+0.8 M_{W_{2}}\left(\frac{R}{1.08}\right)+0 M_{W_{2}}\left(\frac{R}{1.12}\right)=1, \\
0.15 M_{W_{2}}\left(\frac{R}{1.055}\right)+0.7 M_{W_{2}}\left(\frac{R}{1.08}\right)+0.15 M_{W_{2}}\left(\frac{R}{1.12}\right)=1
\end{gathered}
$$

and

$$
0 M_{W_{2}}\left(\frac{R}{1.055}\right)+0.8 M_{W_{2}}\left(\frac{R}{1.08}\right)+0.2 M_{W_{2}}\left(\frac{R}{1.12}\right)=1 .
$$

From the solutions of Equations (5.6), (5.7) and (5.8), this implies $\tilde{R}_{1}(\alpha=0.350000, M=1.103245)=0.614597$.

Other couples $(\alpha, M)$ then $\hat{R}_{0}(\alpha, M), \quad \tilde{R}_{0}(\alpha, M), \quad \hat{R}_{1}(\alpha, M)$ and $\tilde{R}_{1}(\alpha, M)$ are defined as the ones above. Table 5 gives some numerical results of the upper bounds of $\psi^{(1)}(u, \alpha, M), \psi^{(2)}(v, \alpha, M), \phi^{(1)}\left(u, \alpha, M, i_{s}\right)$ and $\phi^{(2)}\left(v, \alpha, M, j_{t}\right)$ for all $s=0,1,2$ and $t=0,1,2$. Note couples $(\alpha, M)$ in Table 5 satisfy Proposition 1.

In Table 5, the upper bounds of (4.14) and (4.15) are tighter than the ones (3.10) and (3.11), respectively. This is in a good accordance with [1] [2]. If $\alpha$ and $M$ increase then the cedent's upper bounds of the ruin probabilities increase while the reinsurer's upper bounds of the ruin probabilities decrease.

\section{Conclusions and Suggestions}

- The surplus processes given by (2.7) and (2.13) can be viewed as an extension of the ones (1.1) and (1.2);

- By martingale method, the author obtains the upper bounds of the ultimate ruin probabilities of the cedent and the reinsurer in the risk models under

\begin{tabular}{|c|c|c|}
\hline & & $\begin{array}{l}\text { Upper bound of } \\
\psi_{n}^{(1)}(u, \alpha, M)\end{array}$ \\
\hline \multicolumn{2}{|c|}{$(\alpha=0.350000, M=1.103245)$} & 0.002537 \\
\hline \multicolumn{2}{|c|}{$(\alpha=0.508604, M=1.903245)$} & 0.005140 \\
\hline \multicolumn{2}{|c|}{$(\alpha=0.758614, M=2.403446)$} & 0.006472 \\
\hline Upper bound of & Upper bound of & Upper bound of \\
\hline$\phi_{n}^{(1)}\left(u, \alpha, M, i_{s}\right)$ & $\psi_{n}^{(2)}(v, \alpha, M)$ & $\phi_{n}^{(2)}\left(v, \alpha, M, j_{t}\right)$ \\
\hline 0.001577 & 0.003103 & 0.001872 \\
\hline 0.003448 & 0.000015 & 0.000006 \\
\hline 0.004417 & 0.000010 & 0.000004 \\
\hline
\end{tabular}
excess of loss reinsurance;

Table 5. The upper bounds of the ruin probabilities for other couples $(\alpha, M)$. 
- There remain many open issues, e.g.

- building the upper bounds of the ultimate ruin probabilities in the risk model under combination of quota share and excess of loss reinsurance;

- investigating the joint ruin probability of the cedent and the reinsurer in the risk model under excess of loss reinsurance;

- establishing the optimality problems under ruin-related optimization criteria. Further research in some of these directions is in progress.

\section{Acknowledgements}

The author wishes to thank professor Bui Khoi Dam for his helpful suggestions and many valuable comments while the author makes this paper.

\section{References}

[1] Yang, H. (2006) Non-Exponential Bounds for Ruin Probability with Interest Effect Included. Scandinavian Actuarial Journal, 1999, 66-79. https://doi.org/10.1080/03461230050131885

[2] Cai, J. and Dickson, D.C.M. (2004) Ruin Probabilities with a Markov Chain Interest Model. Insurance: Mathematics and Economics, 35, 513-525. https://doi.org/10.1016/j.insmatheco.2004.06.004

[3] Centeno, M.L. (1997) Excess of Loss Reinsurance and the Probability of Ruin in Finite Horizon. ASTIN Bulletin, 27, 59-70. https://doi.org/10.2143/AST.27.1.542067

[4] Centeno, M.L. (2002) Measuring the Effects of Reinsurance by the Adjustment Coefficient in the Sparre Anderson Model. Insurance: Mathematics and Economics, 30, 37-49. https://doi.org/10.1016/S0167-6687(01)00095-6

[5] Centeno, M.L. (2002) Excess of Loss Reinsurance and Gerber's Inequality in the Sparre Anderson Model. Insurance: Mathematics and Economics, 31, 415-427. https://doi.org/10.1016/S0167-6687(02)00187-7

[6] Dam, B.K. and Chung, N.Q. (2016) The Martingale Method for Probability of Ultimate Ruin Under Quota $-(\alpha, \beta)$ Reinsurance Model. Journal of Statistics Applications \& Probability, 5, 411-419. https://doi:10.18576/jsap/050305

[7] Dam, B.K. and Chung, N.Q. (2017) On Finite-Time Ruin Probabilities in a Risk Model under Quota Share Reinsurance Contract. Applied Mathematical Sciences, 11, 2609-2629. https://doi.org/10.12988/ams.2017.78240

[8] Kaishev, V.K. and Dimitrova, D.S. (2006) Excess of Loss Reinsurance under Joint Survival Optimality, Insurance: Mathematics and Economics, 39, 376-389. https://doi.org/10.1016/j.insmatheco.2006.05.005

[9] Li, Z. (2008) Optimal Reinsurance Retentions under Ruin-Related Optimization Criteria. Ph. D. Dissertation in Actuarial Science, The University of Waterloo, Ontario, Canada.

[10] Williams, D. (1991) Probability with Martingales. Cambridge University Press, Cambridge. 